\title{
Paraoxonase 1 polymorphisms and ischemic stroke risk: A systematic review and meta-analysis
}

\author{
Issa J. Dahabreh, MD, Georgios D. Kitsios, MD, PhD, David M. Kent, MD, MS, \\ and Thomas A. Trikalinos, $M D, P h D$
}

\begin{abstract}
Purpose: Paraoxonase 1 (PON1) polymorphisms have been implicated as risk factors for coronary artery disease, but the results of genetic association studies on the related phenotype of ischemic stroke are inconclusive. We performed a meta-analysis of published studies investigating the association between ischemic stroke and two nonsynonymous PON1 polymorphisms, rs662 (p.Q192R) and rs854560 (p.L55M) in humans. Methods: We searched multiple electronic databases through June 30, 2009 for eligible studies. In main analyses, we calculated allele-based odds ratios with random effects models. In secondary analyses, we examined dominant and recessive genetic models as well, and performed subgroup and sensitivity analyses. Results: Regarding $r s 662$, we identified 22 eligible studies (total of 7384 cases/ 11,074 controls), yielding a summary odds ratio of 1.10 per $\mathrm{G}$ allele (95\% confidence interval, 1.04-1.17) with no evidence of betweenstudy heterogeneity. For $r s 854560,16$ eligible studies (total of 5518 cases/8951 controls) yielded a summary odds ratio of 0.97 per $\mathrm{T}$ allele (95\% confidence interval, $0.90-1.04$ ), again with no evidence of between-study heterogeneity. For both polymorphisms, analyses with dominant and recessive genetic models yielded the same inferences as allele-based comparisons. Subgroup and sensitivity analyses showed similar results. Conclusion: In agreement with observations in coronary artery disease, PON1 rs662 appears to be associated with a small increase in the risk of ischemic stroke. Genet Med 2010:12(10):606-615.
\end{abstract}

Key Words: paraoxonase 1, PON1, rs662, rs854560, stroke, metaanalysis

$\mathrm{T}$ he paraoxonase 1 (PON1) gene belongs to the paraoxonase gene cluster on 7q21.3-22 and codes for an enzyme with broad substrate specificity. ${ }^{1}$ The PON1 enzyme has lactonase and esterase activity and thus is able to catalyze the hydrolysis of lipid peroxides and organophosphate pesticides. ${ }^{1,2}$ Although its physiologic function has not been fully elucidated, the PON1 enzyme attaches to high-density lipoprotein particles in serum and has been shown to inhibit low-density lipoprotein oxidation, suggesting that PON1 may play a role in atherogenesis. ${ }^{2}$ Two nonsynonymous $P O N 1$ polymorphisms with possible regulatory effects on enzyme activity, ${ }^{2}$ namely $r_{s} 662$ (c.575A $>\mathrm{G}$ or p.Gln192Arg) and $r s 854560$ (c.163T $>$ A or p.Leu55Met) have been extensively investigated as potential risk factors for atherosclerosis-related phenotypes, including coronary artery disease, peripheral arterial disease, and ischemic stroke. ${ }^{2-5}$

From the Institute for Clinical Research and Health Policy Studies, Tufts Medical Center, Boston, Massachusetts.

Thomas Trikalinos, Tufts Medical Center, 800 Washington St, Box 63 , Boston, 02111 MA. E-mail: ttrikalinos@tuftsmedicalcenter.org.

Disclosure: The authors declare no conflict of interest

Submitted for publication January 15, 2010.

Accepted for publication June 15, 2010.

Published online ahead of print September 17, 2010.

DOI: 10.1097/GIM.0b013e3181ee81c6
Two previously published systematic reviews suggested that the $\mathrm{G}$ allele of $r s 662$ is associated with a small increase (perallele odds ratio $[\mathrm{OR}]=1.12$ ) in the risk of coronary artery disease, whereas no such association was found for $r s 854560 .^{4,5}$ Because cerebrovascular and coronary artery disease share many pathophysiologic mechanisms, it is plausible that $r s 662$ could also be a risk factor for ischemic stroke. ${ }^{6-8}$ However, most published studies investigating the relationship between PON1 polymorphisms and ischemic stroke are small in sample size and inconclusive in their results. We therefore performed a meta-analysis to mitigate their shortcomings and summarize the totality of available published evidence on the association between the aforementioned PON1 polymorphisms and ischemic stroke.

\section{METHODS}

\section{Search strategy}

We searched the MEDLINE and SCOPUS databases, and the Human Genome Epidemiology Network Literature Finder (last search June 30th, 2009) to identify English-language studies investigating the association between the PON1 rs662 or $r s 854560$ polymorphisms and ischemic stroke. Search terms included combinations of terms such as "paraoxonase," "PON1," “rs662," “rs854560," "Gln192Arg," "Leu55Met," "Q192R," "L55M," "stroke," "cerebrovascular disease," "cerebral infarction" and their synonyms. The exact search is available on request by the authors. We did not consider other PON1 polymorphisms or polymorphisms in other members of the $P O N$ family of genes (PON2, PON3) because the available evidence on them is limited. We perused the reference lists of all retrieved articles and relevant reviews. We also searched the online archives of Stroke, Annals of Neurology, and Cerebrovascular Diseases, three journals that have published several genetic association studies in ischemic stroke. Eligible studies were those that used case-control, nested case-control, or cohort designs and validated genotyping methods to investigate the frequency of the two polymorphisms in unrelated ischemic stroke patients and unaffected individuals. We did not consider narrative reviews, editorials, and letters to the editor or other articles not reporting primary research results. Family-based studies were excluded because their design and analysis is different from that of population association studies.

\section{Data abstraction}

One investigator abstracted detailed information from each publication regarding study design, matching and ascertainment of controls, demographics, ethnicity of participants (Caucasian continental ancestry, East Asian or other), definition of ischemic stroke, genotyping methods, disease stage, family history, and counts of genotypes and alleles in stroke cases and controls/ unaffected individuals. We relied on the definitions used in each study to subclassify stroke. When studies reported genotype distributions per stroke subtype, we also extracted data for each 
subtype separately, to use in subgroup analyses. We excluded from main analyses studies where patients with ischemic and hemorrhagic stroke were merged together but examined their impact on the meta-analysis results in sensitivity analyses.

\section{Deviations from Hardy-Weinberg equilibrium in the controls}

For each study, we examined whether the distribution of the genotypes in the control group deviated from the Hardy-Weinberg equilibrium (HWE) predicted proportions using an exact test. 9 For studies that did not provide genotype counts, but reported allele frequencies only, we relied on the authors' assessment of deviations from the HWE in the controls.

\section{Meta-analysis}

Main analyses compared allele frequencies of the variant and common allele (G vs. A for $r s 662$ and A vs. T for $r s 854560$ ) between cases and controls. We also evaluated dominant (variant allele carriers versus homozygotes for the common allele) and recessive (homozygotes for the variant allele versus all others) genetic models, for both polymorphisms. We used the OR as a metric of choice. We calculated summary ORs and their 95\% confidence intervals (95\% CI) using the DerSimonian and Laird random effects model. ${ }^{10}$ We tested for between-study heterogeneity with Cochran's $Q$ statistic (considered statistically significant at $P<0.10$ ) and assessed its extent with the $I^{2}$ statistic. ${ }^{11,12} I^{2}$ ranges between 0 and $100 \%$ and expresses the proportion of between study variability that is attributed to heterogeneity rather than chance. Larger $I^{2}$ values imply more extensive heterogeneity.

\section{Subgroup and sensitivity analyses}

We performed prespecified subgroup analyses by source of controls (healthy versus diseased), ethnicity, use of imaging to confirm the diagnosis of stroke, HWE in the control group, and stroke subtype (atherothrombotic versus cardioembolic). We tested for "small-study effects" (differential magnitude of effects in large versus small studies $)^{13}$ with the Harbord modification of the Egger test. ${ }^{14}$ These tests are often erroneously referred to as "publication bias tests." 13,15 We used random effects meta-regression to compare the OR of the first study with the summary OR of subsequent studies or for comparisons between subgroups, as suggested elsewhere. ${ }^{16,17}$ We did not perform adjustments for deviations from the HWE in control genotypes because the necessary genotype counts were not available in a substantial number of studies. ${ }^{9,18}$

\section{Assessing the probability of "false positive" findings}

Associations with $P$-values $<0.05$ are conventionally referred to as "formally statistically significant." Such associations can nevertheless be spurious "false positives," as the end result of chance or bias. Letting biases aside, the probability that a given formally significant result is a "false positive" increases with diminishing prior data in support of the association, decreasing (true) strength of the association, and decreasing statistical power to detect it. ${ }^{19,20}$ We calculated the probability that associations with $P$-values lower than 0.05 are "false" as suggested by Wacholder et al. ${ }^{19,20}$ To this end, we assumed that the true OR was 1.05 (very small effect, similar to those detected in large meta-analyses of genomewide studies), 1.20 or 1.50 (modest effect, expected in most common diseases ${ }^{21}$ ), and that the prior probabilities of genuine association ranged from $10^{-8}$ (a conservative prior, akin to a variant of genomewide significance) to 0.10 (an indicator of strong prior support, appropriate for a functional polymorphism in a gene with strong biological plausibility). ${ }^{20}$ We considered probabilities of "false" association smaller than 0.20 indicative of an important finding.

\section{Software}

Analyses were performed using Stata (version 11/SE, Stata Corp., College Station, TX) and MetaAnalyst (version 3.0 beta, Tufts Medical Center, Boston, MA). ${ }^{22}$ For all tests, except those for heterogeneity, $P$-values were two sided and statistical significance was defined as $P<0.05$. We did not perform any adjustments for multiple comparisons.

\section{RESULTS}

\section{Literature flow}

Our search identified 1040 citations of which 37 were considered potentially eligible and were retrieved in full text. Of those, 19 were excluded (four assessed PON1 activity but not genotypes, nine did not report on ischemic stroke, four were reviews/editorials, and two $\mathrm{o}^{23,24}$ reported on overlapping populations with already included studies). Finally, 22 studies (i.e., 22 independent case-control strata) reported in 17 publications were eligible for the main analyses (Fig. 1). ${ }^{23-39}$

\section{Study characteristics}

Detailed study characteristics are presented in Table 1 . All 22 studies (7384 cases/11,074 controls total) evaluated the rs662 variant and 16 of them (5518 cases/8951 controls total) reported genotyping for the $r s 854560$ variant as well. Sample sizes ranged from 48 to 2092 (median 339). Eleven studies included populations of Caucasian, 10 of East Asian, and 1 of Hispanic descent. For most studies $(n=16)$, there was no information on ischemic stroke subtypes. Three studies reported separate genotype distributions for atherothrombotic and cardioembolic strokes and three included only atherothrombotic strokes. The control groups in four studies for $r s 662$ and one for $r s 854560$ were not in HWE. Notably, only one study reported that genotyping was conducted blinded to the case/control status of participants and no study used genotyping quality-control procedures.

\section{Meta-analysis of rs662}

Figure 2 shows the forest plot for rs662. The summary random effects OR was 1.10 per G allele copy $(95 \% \mathrm{CI}$, $1.04-1.17 ; P=0.001)$, with no evidence for statistical heterogeneity $\left(P_{\mathrm{Q}}=0.12, I^{2}=27 \%\right)$. The association remained significant under a dominant model $(\mathrm{OR}=1.31 ; 95 \% \mathrm{CI}$, $1.05-1.63 ; P=0.02)$ and a recessive model $(\mathrm{OR}=1.22 ; 95 \%$ CI, $1.05-1.43 ; P=0.01)$, with evidence of between-study heterogeneity in the dominant model $(P<0.001$, Table 2$)$. These results remained unchanged in terms of magnitude and significance, when the two additional studies that included a minority of hemorrhagic strokes were also included (24 casecontrol strata, 8008 cases $/ 13,810$ controls; per-allele OR = $1.12 ; 95 \%$ CI, $1.05-1.20 ; P=0.001)$, but there was evidence of between-study heterogeneity $(P=0.02)$.

\section{Meta-analysis of rs854560}

Regarding $r s 854560$, there was no evidence of an association with ischemic stroke risk $(\mathrm{OR}=0.97$ per A allele copy; $95 \%$ CI, 0.90-1.04; $P=0.37$ ) (Fig. 3). Between-study heterogeneity was nonsignificant $\left(P=0.23 ; I^{2}=20 \%\right)$. Alternative genetic models also did not reveal evidence of an association (Table 3 ). 


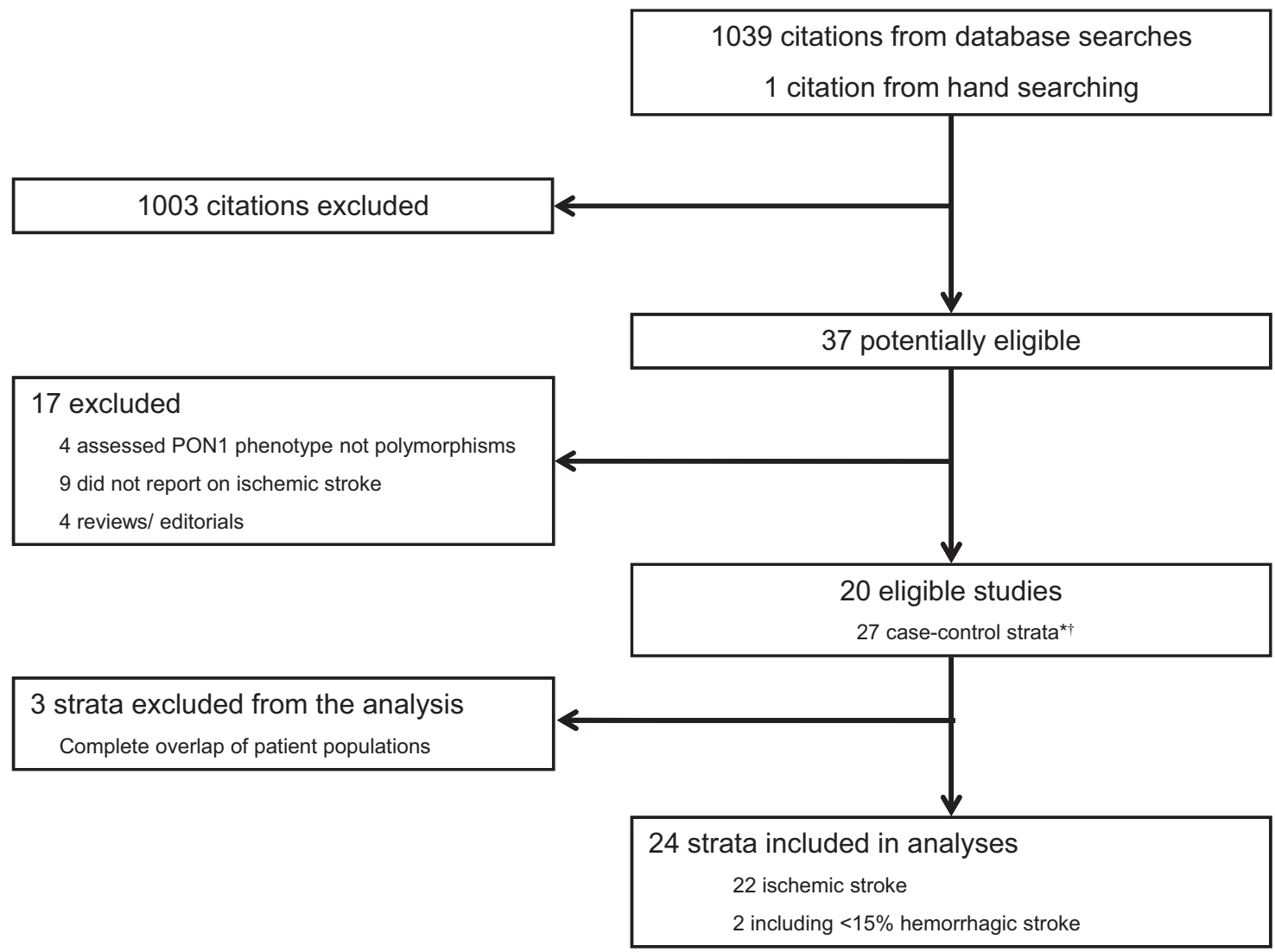

Fig. 1. Literature flow. ${ }^{*} \mathrm{Xu}$ et al. ${ }^{39}$ reported information on five independent case-control groups that were considered as separate "studies" in the analyses. "Wang et al. ${ }^{38}$ reported information on five independent case-control groups that were considered as separate "studies" in the analyses.

Fig. 2. Forest plot of PON1 rs662 and ischemic stroke. Meta-analysis of studies investigating the association of PON1 rs662 with ischemic stroke using a random effects model. Each study is shown by the point estimate of the OR (square proportional to the weight of each study) and $95 \% \mathrm{Cl}$ for the OR (extending lines); the summary OR and $95 \% \mathrm{Cls}$ by random effects calculations is depicted as a diamond. Values higher than 1 indicate that the $G$ allele is associated with increased ischemic stroke risk.

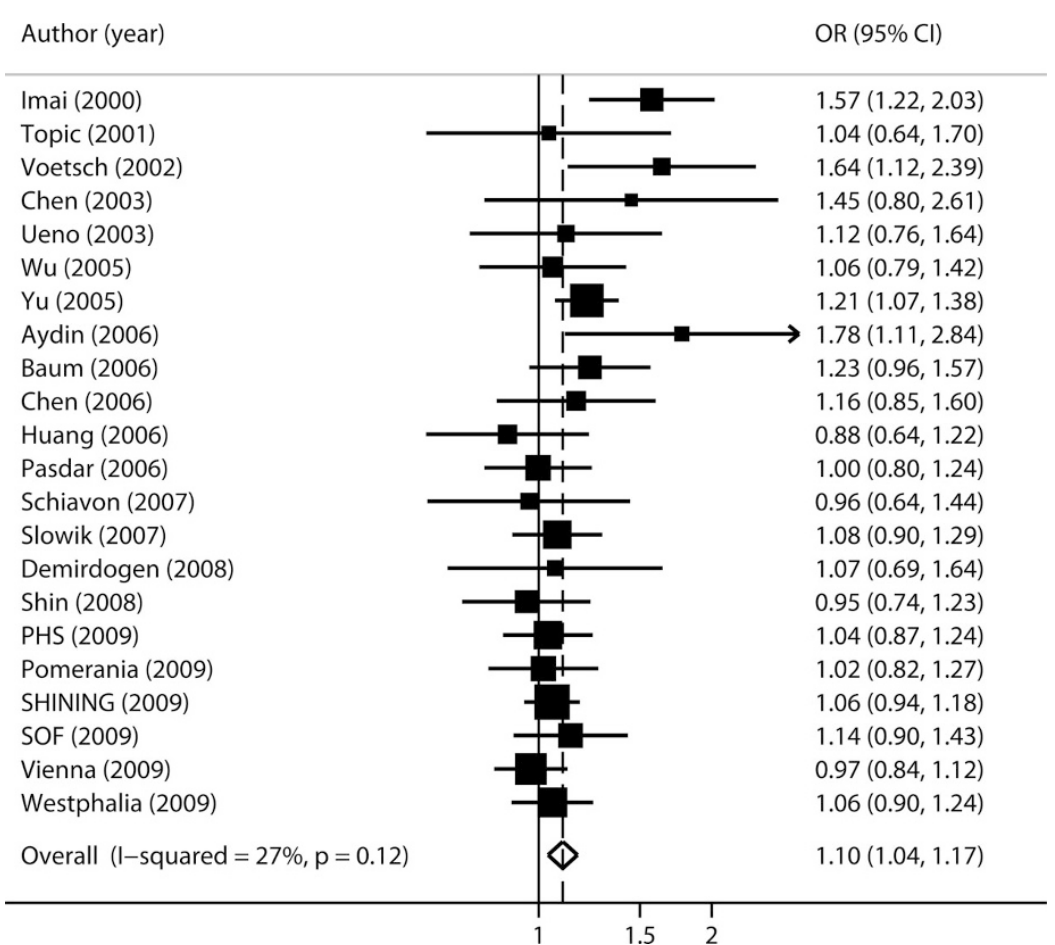




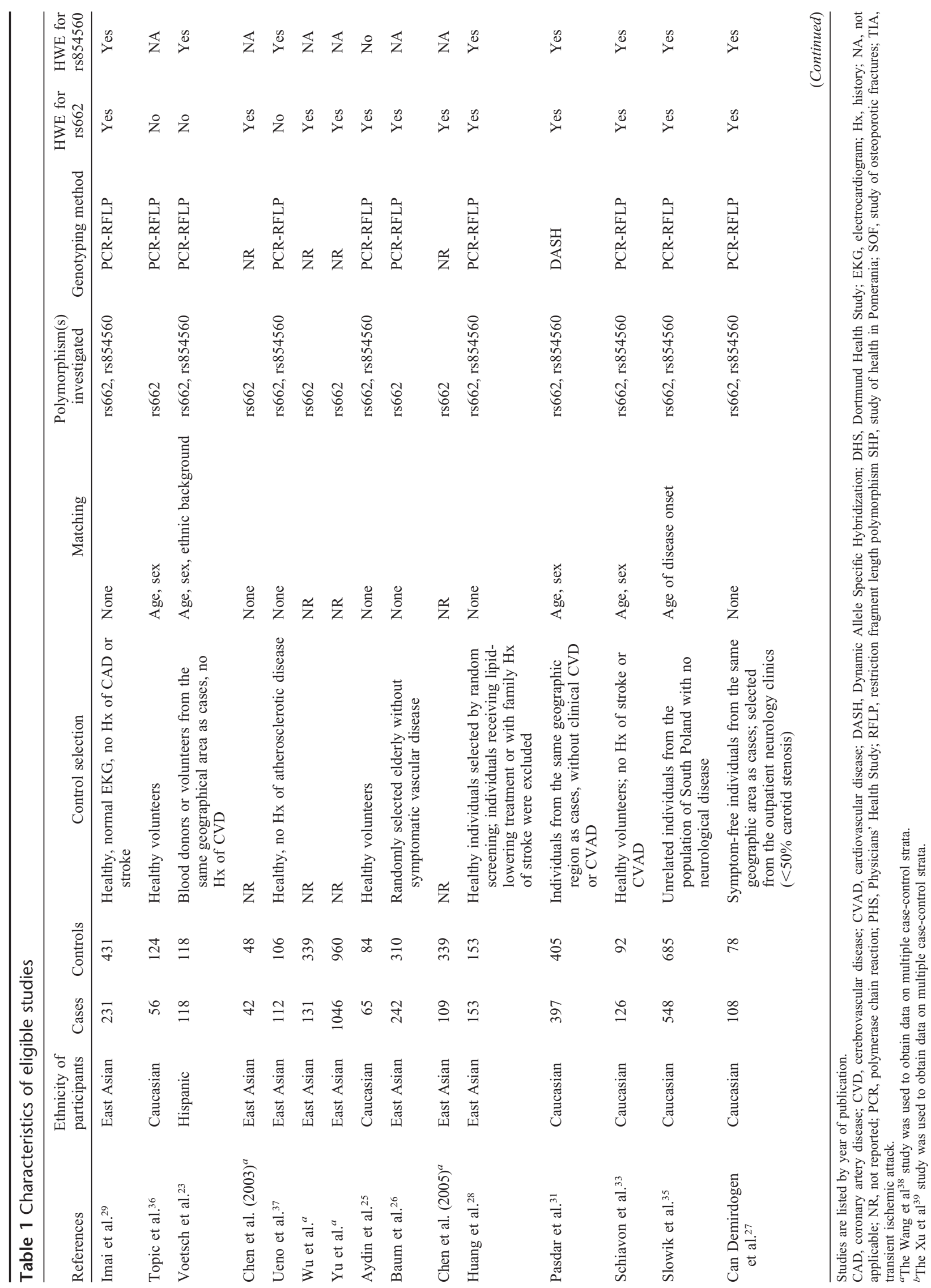




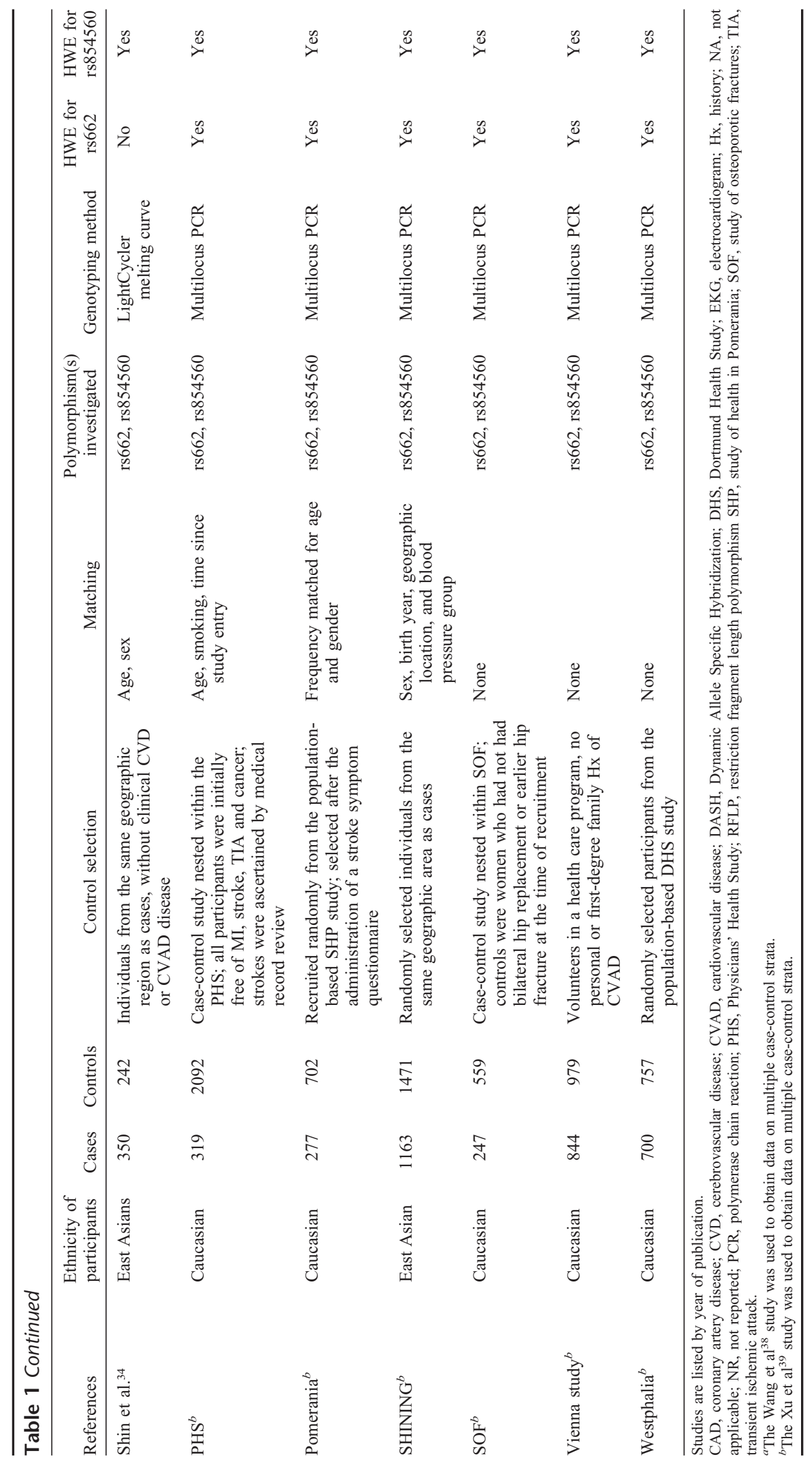




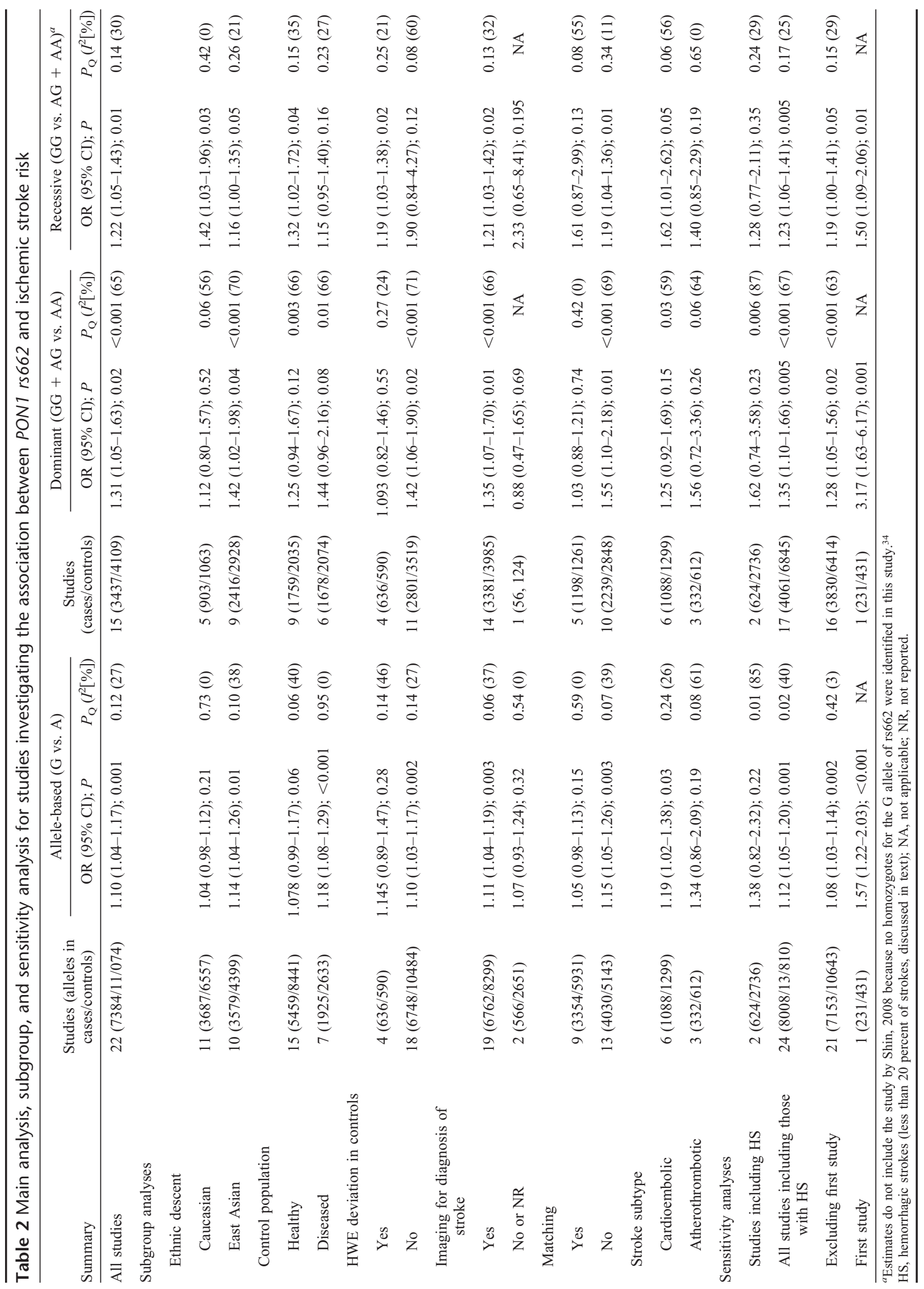


$\begin{array}{ll}\text { Author (year) } & \text { OR }(95 \% \mathrm{Cl})\end{array}$

\begin{tabular}{|c|c|}
\hline Imai (2000) & $0.93(0.60,1.43)$ \\
\hline Voetsch (2002) & $0.98(0.67,1.44)$ \\
\hline Ueno (2003) & $2.78(1.21,6.38)$ \\
\hline Aydin (2006) & $0.68(0.43,1.07)$ \\
\hline Huang (2006) & $0.49(0.17,1.46)$ \\
\hline Pasdar (2006) & $0.96(0.78,1.17)$ \\
\hline Schiavon (2007) & $1.00(0.67,1.51)$ \\
\hline Slowik (2007) & $1.05(0.89,1.24)$ \\
\hline Demirdogen (2008) & $0.76(0.49,1.17)$ \\
\hline Shin (2008) & $0.84(0.50,1.41)$ \\
\hline PHS (2009) & $0.97(0.81,1.15)$ \\
\hline Pomerania (2009) & $1.15(0.94,1.40)$ \\
\hline SHINING (2009) & $1.07(0.83,1.38)$ \\
\hline SOF (2009) & $0.88(0.70,1.10)$ \\
\hline Vienna (2009) & $1.00(0.87,1.14)$ \\
\hline Westphalia (2009) & $0.87(0.75,1.01)$ \\
\hline Overall $(\mid-$ squared $=20 \%, p=0.23)$ & $0.97(0.90,1.04)$ \\
\hline
\end{tabular}

Fig. 3. Forest plot of PON1 rs854560 and ischemic stroke. Meta-analysis of studies investigating the association of PON1 rs854560 with ischemic stroke using a random effects model. Values higher than one indicate that the A allele is associated with increased ischemic stroke risk. Layout similar to Figure 2.

\section{Additional analyses}

Overall, subgroup analysis results were consistent with the main analyses. Tables 4 and 5 present meta-regression analyses for the study-level covariates that we investigated for rs622 and rs854560, respectively. Overall, the factors we explored did not significantly affect the effect size of the genetic associations investigated.

Figure 4 depicts the calculated probability of false association between rs622 and ischemic stroke (not applicable for $r s 854560$ where an association was not found). The figure demonstrates that unless there is a strong prior belief in the association between rs622 and ischemic stroke, the probability that the association is a false positive finding is relatively high, for all possible ORs examined.

There was no evidence that smaller studies had systematically different results compared with larger studies (Harbord test $P=$ 0.14 for rs662 and $P=0.75$ for $r s 854560)$. For $r s 662$, the OR of the first study $(1.57,95 \%$ CI, 1.22-2.03) was statistically significantly different and more extreme compared to the pooled OR of all subsequent studies (interaction test $P=0.005$ ). Omitting the first study reduced between study heterogeneity $\left(P_{\mathrm{Q}}=0.42 ; I^{2}=\right.$ $3 \%)$ and the association remained significant $(\mathrm{OR}=1.08 ; 95 \% \mathrm{CI}$, 1.03-1.14; $P=0.002$ ). Including versus excluding the first study resulted in no appreciable changes for $r s 854560$.

\section{DISCUSSION}

In this systematic review and meta-analysis of epidemiological studies, we found a statistically significant association between the PON1 variant (arginine-encoding, G) allele at $r s 662$ and ischemic stroke. The magnitude of this association is small, as expected for common variants and common diseases. In addition, we found no evidence for an association between $r s 854560$ and the same phenotype. The meta-analyses results were robust in subgroup and sensitivity analyses; however, these analyses may not be powered to detect modest between-subgroup differences because of the relatively small number of studies per subgroup. Although there is a biologically plausible role for PON1 in the pathogenesis of ischemic stroke, we estimated that the probability of the association between rs662 and ischemic stroke being "false-positive" is relatively high for a wide range of assumptions.
The PON1 enzyme attaches to high-density lipoprotein particles and prevents low-density lipoprotein oxidation ${ }^{1}$ and may therefore have a role in the pathophysiology of cardiovascular disease development. ${ }^{40,41}$ There is evidence that polymorphisms in the PON1 gene influence PON1 activity. Rs662 modifies PON1 enzymatic activity in a substrate-dependent manner, and $r s 854560$ is in linkage disequilibrium with functional promoter polymorphisms. ${ }^{41,42}$ Yet, $P O N 1$ polymorphisms explain only a fraction of the variability in PON1 serum activity, $, 2,43,44$ and it is likely that additional genetic and environmental influences contribute to the ischemic stroke phenotype.

The identified association between rs622 and ischemic stroke is consistent with the modest relationship between rs622 and coronary artery disease risk, which has a summary OR of 1.12 per $\mathrm{G}$ allele copy in recent meta-analyses of a different set of studies than those summarized here. ${ }^{4,5}$ Such congruent levels of risk for ischemic stroke and coronary artery disease conferred by the same variant have been also described for other genetic associations. ${ }^{45}$ The fact that these two correlated atherosclerotic phenotypes are associated with the rs622 variant has several alternative explanations.

First, it is possible that the associations of rs662 with both phenotypes are genuine and independent of each other, in which case further laboratory investigation is required to elucidate the underlying mechanisms. Another possible explanation is that the rs622 variant primarily affects an intermediate or surrogate phenotype common to the pathophysiology of both coronary artery disease and ischemic stroke, such as dyslipidemia, ${ }^{34,46}$ increased carotid intima-media thickness, ${ }^{6,46}$ or inflammation. ${ }^{47}$ If this is true, the direct effect of rs622 on the hypothesized intermediate phenotype is expected to be quite larger than the observed associations on the downstream clinical phenotypes of ischemic stroke and coronary artery disease (both around 1.10 per copy of $\mathrm{G}$ allele).

Conversely, it may be that only one of the associations is true or that both are spurious. If only one of the two phenotypes was truly associated with rs622, then the other would also appear to be associated as well, because coronary artery disease and ischemic stroke tend to occur together, i.e., they are correlated. 


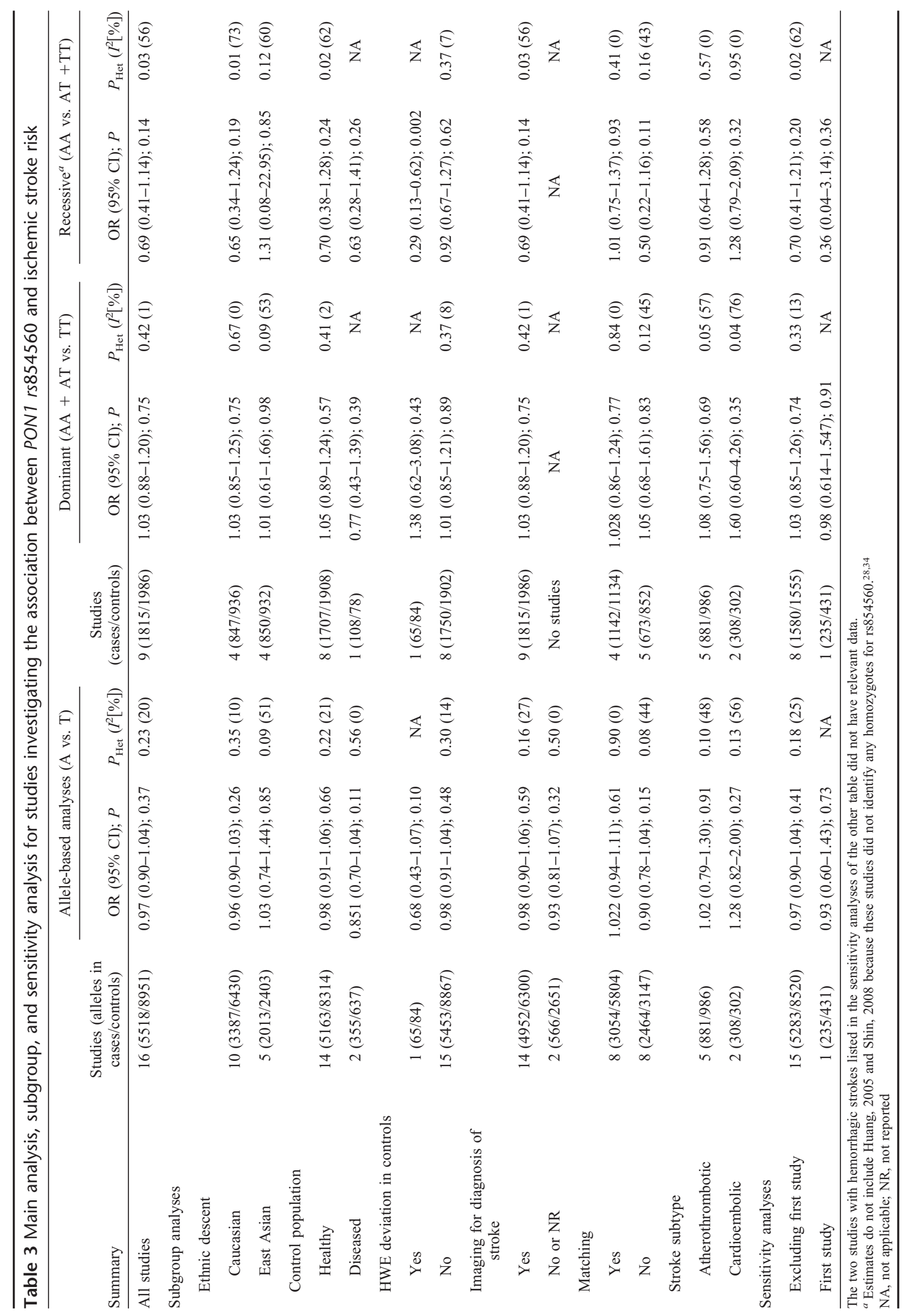


Table 4 Meta-regression results for PON1 rs662

\begin{tabular}{|c|c|c|c|c|c|c|}
\hline \multirow[b]{2}{*}{ Covariate } & \multicolumn{2}{|c|}{ Allele-comparison } & \multicolumn{2}{|c|}{ Dominant } & \multicolumn{2}{|c|}{ Recessive } \\
\hline & Relative OR & Interaction $P$ & Relative OR & Interaction $P$ & Relative OR & Interaction $P$ \\
\hline Ethnicity (East Asians vs. Caucasians) & $1.09(0.98-1.20)$ & 0.12 & $1.25(0.66-2.38)$ & 0.49 & $0.82(0.56-1.19)$ & 0.30 \\
\hline Use of disease controls & $1.11(0.99-1.25)$ & 0.07 & $1.15(0.63-2.10)$ & 0.65 & $0.87(0.63-1.20)$ & 0.40 \\
\hline Control group in HWE & $0.98(0.79-1.20)$ & 0.83 & $1.27(0.68-2.39)$ & 0.46 & $0.74(0.43-1.27)$ & 0.27 \\
\hline Imaging for diagnosis of stroke & $1.03(0.86-1.24)$ & 0.76 & $1.55(0.48-5.00)$ & 0.46 & $1.39(0.66-2.90)$ & 0.39 \\
\hline Matching & $0.92(0.82-1.04)$ & 0.17 & $0.71(0.41-1.22)$ & 0.22 & $1.31(0.85-2.03)$ & 0.23 \\
\hline First study & $1.46(1.12-1.89)$ & 0.005 & $2.61(0.91-7.49)$ & 0.08 & $1.26(0.80-1.97)$ & 0.32 \\
\hline
\end{tabular}

Table 5 Meta-regression results for PON1 rs854560

\begin{tabular}{|c|c|c|c|c|c|c|}
\hline \multirow[b]{2}{*}{ Covariate } & \multicolumn{2}{|c|}{ Allele-comparison } & \multicolumn{2}{|c|}{ Dominant } & \multicolumn{2}{|c|}{ Recessive } \\
\hline & Relative OR & Interaction $P$ & Relative OR & Interaction $P$ & Relative OR & Interaction $P$ \\
\hline Ethnicity (East Asians vs. Caucasians) & $1.07(0.84-1.37)$ & 0.57 & $1.11(0.66-1.88)$ & 0.70 & $1.64(0.19-14.19)$ & 0.65 \\
\hline Use of disease controls & $0.87(0.69-1.09)$ & 0.22 & $0.76(0.43-1.35)$ & 0.36 & $0.90(0.22-3.70)$ & 0.88 \\
\hline Control group in HWE & $1.44(0.87-2.38)$ & 0.15 & $1.48(0.84-2.61)$ & 0.18 & $3.14(1.24-7.96)$ & 0.02 \\
\hline Imaging for diagnosis of stroke & $1.05(0.88-1.25)$ & 0.59 & NA & NA & NA & NA \\
\hline Matching & $1.11(0.98-1.26)$ & 0.09 & $1.20(0.85-1.68)$ & 0.30 & $2.05(0.93-4.55)$ & 0.08 \\
\hline First study & $0.95(0.57-1.58)$ & 0.86 & $0.97(0.52-1.80)$ & 0.92 & $0.51(0.04-6.16)$ & 0.60 \\
\hline
\end{tabular}

Significant results are presented in bold type.

NA, not applicable.

However, in that case, the OR of the phenotype that is not independently associated with the PON1 genotype would be expected to be closer to the null (even for strong betweenphenotype correlations).

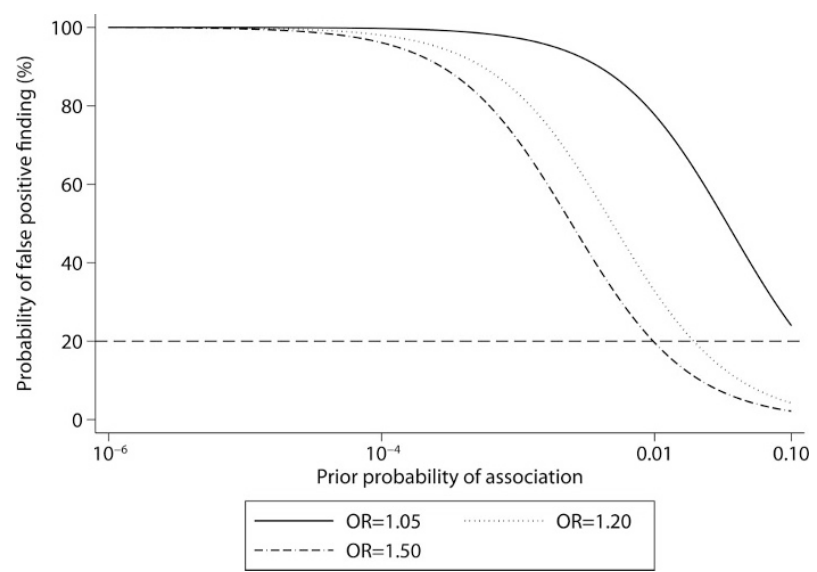

Fig. 4. Probability of "false positive" association between PON1 rs622 and ischemic stroke. Estimation of the probability of "false positive" association between rs622 and ischemic stroke plotted against a wide range of prior probabilities for a genuine association. Calculations as described in Wacholder et al. 2004. ${ }^{20}$ The horizontal dashed line indicates a threshold of a relatively low probability of "false positive" association, operationally set at $20 \%$.
The association of rs662 and ischemic stroke seems to be consistent with the "winner's curse" phenomenon, where the first publication on a gene-disease association reports a spurious or exaggerated effect size that is not replicated by subsequent research. ${ }^{16}$ "Winner's curse" may be the result of various selection biases, such as time-lag bias, shortcomings in the design, and conduct of individual studies, or chance. Briefly, time lag bias exists when the order of study publication depends on study results, e.g., with statistically significant studies being published first, and nonsignificant studies published subsequently. In its extreme form, nonsignificant studies remain unpublished (publication bias). Publication bias likely exists in genetic and genomic topics but cannot be measured directly. This is because most of the so-called "publication bias diagnostics" simply test for systematic differences between more and less precise studies, for which publication bias is only one of many possible explanations. ${ }^{13,15}$

Further, although there are no validated quality characteristics to distinguish association studies with higher versus lower risk of bias, one can use criteria with substantial face validity. In our topic, only one study explicitly mentioned blinding of investigators to the case/control status of participants, no studies reported using genotyping quality control procedures, and in several studies, the genotypic frequencies in the control groups deviated from those expected under HWE. Finally, as shown in Figure 4 , the probability that the association is due to chance ("falsely" positive) is relatively high for a wide range of assumptions. It remains to be defined whether functional evidence on rs622 and pathophysiological evidence implicating PON1 in ischemic stroke are supportive of strong biological plausibility. In such case, further studies would be required to disentangle 
the mechanistic effects of this genetic variant and confirm the findings of our meta-analysis.

In conclusion, we found evidence of a weak association between $r s 662$ and ischemic stroke risk, similar in magnitude to the corresponding association of the variant with coronary disease. Genetic variation in the paraoxonase gene cluster merits further investigation, preferably using haplotyping approaches to comprehensively assess its relationship with atherosclerotic disease risk and elucidate the molecular basis of the observed genetic effects.

\section{ACKNOWLEDGMENTS}

This project was partially supported by Award Number UL1RR025752 from the National Center for Research Resources. The content is solely the responsibility of the authors and does not necessarily represent the official views of the National Center for Research Resources or the National Institutes of Health. IJD is the recipient of a research fellowship provided by the "Maria P. Lemos" Foundation. GDK is a recipient of a Pfizer-Tufts Medical Center career development award.

\section{REFERENCES}

1. Mackness MI, Arrol S, Durrington PN. Paraoxonase prevents accumulation of lipoperoxides in low-density lipoprotein. FEBS Lett 1991;286:152-154.

2. Mackness M, Mackness B. Paraoxonase 1 and atherosclerosis: is the gene or the protein more important? Free Radic Biol Med 2004;37:1317-1323.

3. Roest M, Jansen AC, Barendrecht A, Leus FR, Kastelein JJ, Voorbij HA. Variation at the paraoxonase gene locus contributes to carotid arterial wall thickness in subjects with familial hypercholesterolemia. Clin Biochem 2005;38:123-127.

4. Wheeler JG, Keavney BD, Watkins H, Collins R, Danesh J. Four paraoxonase gene polymorphisms in 11212 cases of coronary heart disease and 12786 controls: meta-analysis of 43 studies. Lancet 2004;363:689-695.

5. Lawlor DA, Day IN, Gaunt TR, et al. The association of the PON1 Q192R polymorphism with coronary heart disease: findings from the British Women's Heart and Health cohort study and a meta-analysis. BMC Genet 2004;5:17.

6. Humphries SE, Morgan L. Genetic risk factors for stroke and carotid atherosclerosis: insights into pathophysiology from candidate gene approaches. Lancet Neurol 2004;3:227-235.

7. Morrison AC, Bare LA, Luke MM, et al. Single nucleotide polymorphisms associated with coronary heart disease predict incident ischemic stroke in the atherosclerosis risk in communities study. Cerebrovasc Dis 2008;26:420-424.

8. Luke MM, Lalouschek W, Rowland CM, et al. Polymorphisms associated with both noncardioembolic stroke and coronary heart disease: Vienna stroke registry. Cerebrovasc Dis 2009;28:499-504.

9. Trikalinos TA, Salanti G, Khoury MJ, Ioannidis JP. Impact of violations and deviations in Hardy-Weinberg equilibrium on postulated gene-disease associations. Am J Epidemiol 2006;163:300-309.

10. DerSimonian R, Laird N. Meta-analysis in clinical trials. Control Clin Trials 1986; 7:177-188.

11. Cochran W. The combination of estimates from different experiments. Biometrics 1954;10:101-129.

12. Higgins JP, Thompson SG. Quantifying heterogeneity in a meta-analysis. Stat Med 2002;21:1539-1558.

13. Lau J, Ioannidis JP, Terrin N, Schmid CH, Olkin I. The case of the misleading funnel plot. BMJ 2006;333:597-600

14. Harbord RM, Egger M, Sterne JA. A modified test for small-study effects in meta-analyses of controlled trials with binary endpoints. Stat Med 2006;25: 3443-3457.

15. Terrin N, Schmid CH, Lau J. In an empirical evaluation of the funnel plot, researchers could not visually identify publication bias. $J$ Clin Epidemiol 2005;58:894-901.

16. Ioannidis JP, Ntzani EE, Trikalinos TA, Contopoulos-Ioannidis DG. Replication validity of genetic association studies. Nat Genet 2001;29:306-309.

17. Knapp G, Hartung J. Improved tests for a random effects meta-regression with a single covariate. Stat Med 2003;22:2693-2710.

18. Salanti G, Higgins JP, Trikalinos TA, Ioannidis JP. Bayesian meta-analysis and meta-regression for gene-disease associations and deviations from Hardy-Weinberg equilibrium. Stat Med 2007;26:553-567.

19. Thomas DC, Clayton DG. Betting odds and genetic associations. J Nat Cancer Inst 2004;96:421-423.

20. Wacholder S, Chanock S, Garcia-Closas M, El Ghormli L, Rothman N. Assessing the probability that a positive report is false: an approach for molecular epidemiology studies. J Natl Cancer Inst 2004;96:434-442.

21. Ioannidis JP, Trikalinos TA, Khoury MJ. Implications of small effect sizes of individual genetic variants on the design and interpretation of genetic association studies of complex diseases. Am J Epidemiol 2006;164:609-614.

22. Wallace BC, Schmid CH, Lau J, Trikalinos TA. Meta-Analyst: software for meta-analysis of binary, continuous and diagnostic data. BMC Med Res Methodol 2009;9:80.

23. Voetsch B, Benke KS, Damasceno BP, Siqueira LH, Loscalzo J. Paraoxonase $192 \mathrm{Gln}-\gg$ Arg polymorphism: an independent risk factor for nonfatal arterial ischemic stroke among young adults. Stroke 2002;33:1459-1464.

24. Voetsch B, Benke KS, Panhuysen CI, Damasceno BP, Loscalzo J. The combined effect of paraoxonase promoter and coding region polymorphisms on the risk of arterial ischemic stroke among young adults. Arch Neurol 2004;61:351-356.

25. Aydin M, Gencer M, Cetinkaya Y, et al. PON1 55/192 polymorphism, oxidative stress, type, prognosis and severity of stroke. IUBMB Life 2006; 58:165-172

26. Baum L, Ng HK, Woo KS, et al. Paraoxonase 1 gene Q192R polymorphism affects stroke and myocardial infarction risk. Clin Biochem 2006;39:191-195.

27. Can Demirdogen B, Turkanoglu A, Bek S, et al. Paraoxonase/arylesterase ratio, PON1 192Q/R polymorphism and PON1 status are associated with increased risk of ischemic stroke. Clin Biochem 2008;41:1-9.

28. Huang Q, Liu YH, Yang QD, et al. Human serum paraoxonase gene polymorphisms, Q192R and L55M, are not associated with the risk of cerebral infarction in Chinese Han population. Neurol Res 2006;28:549-554.

29. Imai Y, Morita H, Kurihara H, et al. Evidence for association between paraoxonase gene polymorphisms and atherosclerotic diseases. Atherosclerosis 2000;149:435-442.

30. Lansbury AJ, Catto AJ, Carter AM, Bamford JM, Grant PJ. The paraoxonase glutamine/arginine polymorphism and cerebrovascular disease. Cerebrovasc Dis 2004; 7:353-355

31. Pasdar A, Ross-Adams H, Cumming A, et al. Paraoxonase gene polymorphisms and haplotype analysis in a stroke population. BMC Med Genet 2006;7:28

32. Ranade K, Kirchgessner TG, Iakoubova OA, et al. Evaluation of the paraoxonases as candidate genes for stroke: Gln192Arg polymorphism in the paraoxonase 1 gene is associated with increased risk of stroke. Stroke 2005;36:2346-2350.

33. Schiavon R, Turazzini M, De Fanti E, et al. PON1 activity and genotype in patients with arterial ischemic stroke and in healthy individuals. Acta Neurol Scand 2007;116:26-30.

34. Shin BS, Oh SY, Kim YS, Kim KW. The paraoxonase gene polymorphism in stroke patients and lipid profile. Acta Neurol Scand 2008;117:237-243.

35. Slowik A, Wloch D, Szermer P, et al. Paraoxonase 2 gene $\mathrm{C} 311 \mathrm{~S}$ polymorphism is associated with a risk of large vessel disease stroke in a Polish population. Cerebrovasc Dis 2007;23:395-400.

36. Topic E, Simundic AM, Ttefanovic M, et al. Polymorphism of apoprotein E (APOE), methylenetetrahydrofolate reductase (MTHFR) and paraoxonase (PON1) genes in patients with cerebrovascular disease. Clin Chem Lab Med 2001;39:346-350

37. Ueno T, Shimazaki E, Matsumoto T, et al. Paraoxonase1 polymorphism Leu-Met55 is associated with cerebral infarction in Japanese population. Med Sci Monit 2003;9:CR208-CR212.

38. Wang X, Cheng S, Brophy VH, et al. A meta-analysis of candidate gene polymorphisms and ischemic stroke in 6 study populations: association of lymphotoxin-alpha in nonhypertensive patients. Stroke 2009;40:683-695.

39. Xu X, Li J, Sheng W, Liu L. Meta-analysis of genetic studies from journals published in China of ischemic stroke in the Han Chinese population. Cerebrovasc Dis 2008;26:48-62.

40. Jarvik GP, Rozek LS, Brophy VH, et al. Paraoxonase (PON1) phenotype is a better predictor of vascular disease than is PON1(192) or PON1(55) genotype. Arterioscler Thromb Vasc Biol 2000;20:2441-2447.

41. Brophy VH, Jampsa RL, Clendenning JB, McKinstry LA, Jarvik GP, Furlong CE. Effects of $5^{\prime}$ regulatory-region polymorphisms on paraoxonasegene (PON1) expression. Am J Hum Genet 2001;68:1428-1436.

42. Chen J, Chan W, Wallenstein S, Berkowitz G, Wetmur JG. Haplotypephenotype relationships of paraoxonase-1. Cancer Epidemiol Biomarkers Prev 2005;14:731-734.

43. Thyagarajan B, Jacobs DR Jr, Carr JJ, et al. Factors associated with paraoxonase genotypes and activity in a diverse, young, healthy population: the Coronary Artery Risk Development in Young Adults (CARDIA) study. Clin Chem 2008;54:738-746.

44. Rainwater DL, Rutherford S, Dyer TD, et al. Determinants of variation in human serum paraoxonase activity. Heredity 2009;102:147-154.

45. Bentley P, Peck G, Smeeth L, Whittaker J, Sharma P. Causal relationship of susceptibility genes to ischemic stroke: comparison to ischemic heart disease and biochemical determinants. PLoS One 2010;5:e9136.

46. Carlson CS, Heagerty PJ, Hatsukami TS, et al. TagSNP analyses of the PON gene cluster: effects on PON1 activity, LDL oxidative susceptibility, and vascular disease. J Lipid Res 2006;47:1014-1024.

47. Fan AZ, Yesupriya A, Chang MH, et al. Gene polymorphisms in association with emerging cardiovascular risk markers in adult women. BMC Med Genet 2010;11:6 